ARTICLE

\title{
Exclusive manual perineal rehabilitation with lidocaine $2 \%$ gel in the treatment of provoked vestibulodynia: results from a single-arm interventional study
}

\author{
A. Close $(i)^{1,2 \bowtie}$, M. G. Culha iD $^{3}$, V. Albert ${ }^{2,4}$ and G. Valancogne $e^{5}$ \\ (c) The Author(s), under exclusive licence to Springer Nature Limited 2022
}

As of now, there is no adequate therapeutic strategy for provoked vestibulodynia (PVD). Pelvic Floor Muscle Therapy (PFMT) is a widely used technique in general pelvic floor rehabilitation. The objective of this study is to examine the effects of exclusive manual perineal rehabilitation with lidocaine $2 \%$ gel on PVD. During the first session, recruited patients $(n=68$; mean age $31 \pm 8.6$; range: 18-52) received a questionnaire (Q1) on general well-being and health, pain of the genital area, sexual function, and symptoms during vaginal penetration. This questionnaire was based on a generalised questionnaire on the quality of life, the Medical Outcomes Study 36-item (SF-36), the Female Sexual Function Index (FSFI), and the Visual Analogue Scale (VAS). A second identical questionnaire with an additional set of open-ended questions concerning the assessment of the treatment was collected after treatment (Q2). A total of 45 questionnaires were completed. Statistical results showed a significant improvement of all items before and after treatment $(p<0.001)$ : perceived general well-being and health, perceived vulvar pain, perceived sexual function, and perceived vaginal penetration. In conclusion, exclusive manual perineal rehabilitation using lidocaine $2 \%$ gel seems to be a safe and effective treatment option for vulvodynia in women.

IJIR: Your Sexual Medicine Journal (2023) 35:157-163; https://doi.org/10.1038/s41443-022-00537-9

\section{INTRODUCTION}

Vulvodynia is defined by the International Society for the Study of Vulvovaginal Disease as chronic pain or discomfort in the area of the vulva for more than three months, with no identifiable aetiological cause [1]. Despite its prevalence, it is poorly known among healthcare professionals and is currently underdiagnosed and under-treated [2-5]. Lifetime prevalence is estimated at $3-14 \%$ in women of all ethnicities, with a mean age of onset of 30 [3-6].

Generalised vulvodynia concerns the whole vulva, but pain may be localised specifically on the clitoris (clitorodynia) or the vestibule of the vagina (vestibulodynia) [1]. Provoked vestibulodynia (PVD) may be induced by contact (cotton-swab, tampon, penetration), while unprovoked vulvodynia is characterised by pain without any trigger. Women can present with mixed vulvodynia [1]. Likewise, primary vulvodynia, where pain is reported since the first attempt of tampon insertion or sexual penetration, can be distinguished from secondary vulvodynia, which arises after a period of more than 3 months of pain-free intercourse [1, 7]. Temporal patterns (intermittent, persistent, constant, immediate, or delayed) are described $[1,8]$.

The impact of PVD is significant, causing dyspareunia and avoidance of sexual activities, which can seriously affect relationships and quality of life as well as lead to other psychological alterations such as anxiety and depression [9-12].
In the literature, information about idiopathic vulvodynia therapy is limited, and no consensus has been reached [13]. De Andres et al. stated that the optimal therapy for vulvar pain syndrome remains elusive, with low percentages of therapeutic success, especially using local or systemic pharmacological approaches [14]. Morin et al. concluded in their systematic review that physical therapy techniques such as biofeedback, dilators, education, electrical stimulation, multimodal physical therapy, and multidisciplinary approaches were effective in decreasing pain during intercourse and improving sexual function [15].

The aetiology of PVD is hypothesised to be multifactorial with an interdependency on biopsychosocial factors [16-19]. The involvement of the pelvic floor muscles (PFMs) is well known and has been demonstrated in several controlled studies [20-24]. Women with PVD show raised resting PFM tone, reduced coordination, strength and flexibility, impaired voluntary relaxation, and decreased endurance [21, 23, 24]. Several clinical guidelines have defined PFMT as a first-line treatment of PVD $[2,19,25]$.

However, Morin et al. [15] concluded in their systematic review that there were no studies evaluating manual therapy as an isolated modality in the selected population. Given the lack of a precise and detailed physiotherapeutic treatment protocol for PVD, this study aims to investigate the effects of exclusive manual perineal rehabilitation with lidocaine $2 \%$ gel on PVD. Additionally,

\footnotetext{
${ }^{1}$ Department of Physiotherapy, Pelvi-Perineal Rehabilitation Section, Clinique St Jean, Boulevard du Jardin Botanique 32, 1000 Brussels, Belgium. ${ }^{2}$ Medicis Medical Center, Avenue de Tervueren 236, 1150 Brussels, Belgium. ${ }^{3}$ Department of Urology, University of Health Sciences, Prof. Dr. Cemil Tescioglu City Hospital, Istanbul, Turkey. ${ }^{4}$ Department of Gynecology and Obstetrics, CHU St Pierre, Rue aux Laines 105, 1000 Brussels, Belgium. ${ }^{5}$ Avenue Maréchal de Saxe 106, 69003 Lyon, France. ${ }^{凶}$ email: aclose@clstjean.be
}

Received: 26 September 2021 Revised: 27 January 2022 Accepted: 1 February 2022

Published online: 28 February 2022 
158

the study aimed to evaluate the sexual function and pain perception of the patients, the degree of pain before, during and after vaginal penetration, and the type of pain.

\section{POPULATION AND METHOD}

The present cohort study is an interventional multicentric study conducted between March 2019 and December 2020. Protocol of the study was approved by the Ethical Committee of ErasmeUniversité Libre de Bruxelles (Belgium; B07601939478). Informed consent of all patients was obtained. The patients were recruited in the pelvic floor departments of the Clinique Saint Jean and the Medicis Medical Center, Brussels.

Women presenting with symptoms of vulvar pain at the outpatient clinic and subsequently diagnosed with PVD $(n=68)$ received a questionnaire at the first session of physiotherapy (Q1) from the physiotherapist experimenter. Patients underwent perineal rehabilitation at least once a week. The same questionnaire was presented at the end of the treatment with openended questions about perceived improvement as a result of the treatment (Q2).

\section{Sample and recruitment}

Patients had to meet the following inclusion criteria to be eligible to participate in this study: a positive cotton-swab result (which is the standard gynaecological method for diagnosing PVD) $[2,19]$, vulvodynia persisting for $>3$ months, defined as experiencing pain on a daily basis, 18-65 years of age, and signed consent form. The exclusion criteria could be defined according to the recommendations of the guidelines concerning the treatment of vulvodynia $[19,25]$ : other ongoing therapeutic treatment (pain medication, anti-inflammatory drugs, antidepressants, anticonvulsants, topical vulvovaginal cream, oestrogen cream, injection of dermocorticoids, lidocaine of botulinum toxin), clinically significant dermatological disease such as eczema, lichen sclerosus and planus, psoriasis and clinically significant neurological disease such as pudendal neuralgia.

Based on the data in the study of Glazer et al. [24], it was decided to include at least 42 patients in the study, with a power of $80 \%$ and a margin of error of $5 \%$.

\section{Population}

A questionnaire and a protocol of treatment were established for this study in French, Dutch, and English. The questionnaire was based on validated questionnaires, the SF-36 and the FSFI, and on the VAS. The SF-36 relates to perceived general health and is recommended as a self-report outcome measure in vulvodynia clinical trials [26]. The FSFI is the most commonly used index for assessing sexual function in women with vulvar pain $[27,28]$. The VAS has well-established reliability and validity across general pain populations [28]. Vulvar pain was quantified using a $10 \mathrm{~cm}$ linear VAS (with $0=$ no pain and $10=$ maximum pain).

The questions were subdivided into four different items: general well-being and health, pain of the genital area, sexual function, and vaginal penetration. Some open-ended questions relating to the perceived improvement as a result of physiotherapy treatment were added in the Q2. Multiple-choice closed questions are scored from 0 to 4 points, while closed dichotomous questions are scored from 0 or 1 point. Each item represents one score: (i) score on 17 points for the general well-being and health item; (ii) 25 points for the pain of genital area item; (iii) 40 points for the sexual function item; and (iv) 71 points for the vaginal intercourse item. The final score is the sum of the scores of all the items. The maximum score that can be achieved is 153 ( $153=$ negative global feeling; $0=$ positive global feeling). Open-ended questions did not require a score since they reported each participant's personal opinion of the treatment.
A description of the pelvic floor's anatomy and physiopathology was given by the caregiver. It is essential to begin treatment with an explanation of the disease and determine realistic treatment goals $[19,25]$.

According to Bergeron et al. [29], physical therapy interventions aim at rehabilitating the PFMs by (i) increasing PFMs awareness and proprioception; (ii) improving PFMs dissociation and relaxation; (iii) normalising PFMs tone; (iv) increasing elasticity of the muscle and vaginal tissues; and (v) reducing fear of vaginal penetration $[15,29]$. The authors of the present study added some specifications to their protocol: (vi) desensitising the painful area with lidocaine $2 \%$ gel; (vii) teaching the practice of self-massage at home; (viii) decreasing apprehension of vaginal penetration by using vaginal dilators of increased diameters with breathing techniques; (ix) counselling about lifestyle and personal hygiene, and $(x)$ coaching about sexuality. The same therapist carried out all sessions lasting $30 \mathrm{~min}$ at two different centres. Due to the variability of progress, an individual treatment was preferred.

The vulvar pain is often localised between 4 and 8 o'clock on the introitus, just exterior to the hymenal ring [30]. Technique was achieved through half a phalanx of a single finger insertion to the painful spot with lidocaine $2 \%$ gel. Depending of the peak points within pain-related regions, delicate pressure-massage was carried out at the 5, 6 and 7 o'clock positions of the vestibule, the entire vestibule, and/or on the clitoris. Patients were advised to perform the same massage at home twice a day. Some preceding findings exhibited a significantly lower pressure-pain threshold in women with PVD than control women [31, 32]. Others provide further evidence of allodynia in women with PVD, finding that control women perceived a touch sensation at levels of pressure that were already painful for women with PVD [32].

Delicate genital hygiene is recommended such as use of clean water (no vulvar irritating agents), cotton underwear, cotton sanitary pads, and a good lubricant for sexual intercourse $[2,25,33]$.

\section{Statistical analysis}

The data collected with the questionnaires were analysed using JASP system (version 0.13.0.0). To begin with, the normality of the variables was evaluated by Shapiro-Wilk's test statistics. Because the $p$-value was above 0.05 , the variable was considered normal. Next, two different statistical tests were used to compare the data before and after treatment: (i) the parametric test of $t$-Student to analyse the data of the general, well-being and health, painful, and sexual function items; (ii) the nonparametric Wilcoxon signedranks test for paired sample to assess the VAS before, during and after vaginal intercourse. For all analyses, a $p$ value of less than 0.05 was set as statistically significant.

\section{RESULTS}

Overall, 68 women, aged 18-65 years, diagnosed with objective vulvodynia, defined as vulvar pain for at least 3 months in the past 6 months, engaged in the study. On this panel, 45 women $(66 \%)$ filled out both the questionnaires, and 23 (34\%) were excluded due to exclusion criteria, lack of compliance of therapy, and failure to follow up due to the COVID-19 pandemic. Among the patients, $40 \%(n=18)$ were diagnosed by a gynaecologist, $58 \%(n=26)$ by the experimenter and researcher (AC), and only one by $a$ dermatologist $(2 \%)$. In all, $58 \%(n=26)$ of the study sample suffered from primary vulvodynia and $42 \%(n=19)$ experienced secondary vulvodynia. Primary vaginismus was found in $22 \%$ of the patients $(n=10)$, localised PVD in 91\% $(n=41)$, and generalised PVD in the rest of the sample $(n=4)$ (Table 1). Relating to psychological comorbidities, the most widespread complaint was anxiety with $62 \%$ before treatment $(n=28)$ and $40 \%$ after $(n=$ 18). Thirteen women (29\%) presented no psychological affections 
before treatment compared to 23 after treatment (51\%) (Table 2). The average number of physiotherapy sessions was 9.67 (SD \pm 3.88; range 4-20) for a duration of $30 \mathrm{~min}$ and a period of treatment of 2.78 months ( $S D \pm 2.5$; range $1-6$ ) (Table 1 ).

Table 1. General data

\begin{tabular}{llll|} 
& Mean & s.d. & Range \\
\hline Age & 31 & 8.6 & $18-52$ \\
\hline Primary PVD & $N$ & $\%$ & \\
\hline Secundary PVD & 26 & 58 & \\
\hline Localised PVD & 19 & 42 & \\
\hline Generalised PVD & 42 & 93 & \\
\hline Primary vaginismus & 3 & 7 & \\
\hline Diagnosed by the experimenter & 26 & 58 & \\
\hline Diagnosed by a gynaecologist & 18 & 40 & \\
\hline Diagnosed by a dermatologist & 1 & 2 & \\
\hline Number of sessions & 9.67 & 3.88 & $4-20$ \\
\hline Period of treatment (months) & 2.78 & 2.5 & $1-6$ \\
\hline
\end{tabular}

Perceived global score per item

A significant difference in the perceived global score at the start of treatment compared to the end of treatment was demonstrated $(p<0.001)$. The scores of all items before and after treatment were significantly improved $(p<0.001)$ : perceived general well-being and health, perceived vulvar pain, perceived sexual function and perceived vaginal penetration (Table 2; Fig. 1).

\section{Pain evaluation and characteristics}

A statistically significant positive evolution is shown for perceived genital pain before, during, and after intercourse through the VAS score (mean 3.11-1.21; 7.70-4.67; 5.78-2.52, respectively; $p<0.001$ ) (Table 2 ). Burning, stinging, irritation, stabbing, and rawness are the symptoms described in the literature [1, 19,33]. In this study, the most pronounced pain characteristics at the beginning of treatment were burning ( $n=$ $35 ; 78 \%)$ and then stabbing $(n=24 ; 53 \%)$. At the end of treatment, $67 \%$ of the sample presented burning $(n=30)$ and $40 \%$ experienced tugging $(n=18)$ (Table 2$)$.

\section{Self-massage score}

Self-massage of the painful area using lidocaine $2 \%$ gel was reported by 38 women (84\%), out of which 26 individuals applied it every day (58\%). Frequency varied from once a week $(4 \%)$ to

Table 2. Comparisons of patients between pre and posttreatment.

\begin{tabular}{|c|c|c|c|c|c|c|}
\hline & \multicolumn{2}{|c|}{ Before treatment } & \multicolumn{2}{|c|}{ After treatment } & & \\
\hline & $N$ & $\%$ & $N$ & $\%$ & & \\
\hline \multicolumn{7}{|l|}{ Psychological comorbidities } \\
\hline Depressed & 6 & 13 & 2 & 4 & & \\
\hline Sad & 8 & 18 & 2 & 4 & & \\
\hline None & 13 & 29 & 23 & 51 & & \\
\hline \multicolumn{7}{|l|}{ Score per items } \\
\hline Perceived well-being and health & 6.22 & 2.86 & 4.64 & 2.26 & $<0.001$ & $-25 \%$ \\
\hline Perceived vulvar pain & 8.91 & 3.32 & 5.71 & 3.36 & $<0.001$ & $-36 \%$ \\
\hline Perceived sexual function & 21.60 & 6.76 & 17.78 & 7.10 & $<0.001$ & $-18 \%$ \\
\hline Perceived vaginal penetration & 40.05 & 12.40 & 29.17 & 12.99 & $<0.001$ & $-27 \%$ \\
\hline During vaginal penetration & 7.70 & 2.01 & 4.67 & 2.88 & $<0.001$ & $-39 \%$ \\
\hline \multirow[t]{2}{*}{ After vaginal penetration } & 5.78 & 2.75 & 2.52 & 2.41 & $<0.001$ & $-56 \%$ \\
\hline & $N$ & $\%$ & $N$ & $\%$ & & \\
\hline \multicolumn{7}{|l|}{ Characteristics of pain } \\
\hline Burning & 35 & 78 & 30 & 67 & & \\
\hline Stabbing & 24 & 53 & 14 & 31 & & \\
\hline Tugging & 22 & 49 & 18 & 40 & & \\
\hline Tingling & 20 & 44 & 14 & 31 & & \\
\hline Pinch & 19 & 42 & 15 & 33 & & \\
\hline Rawness & 19 & 42 & 14 & 31 & & \\
\hline
\end{tabular}



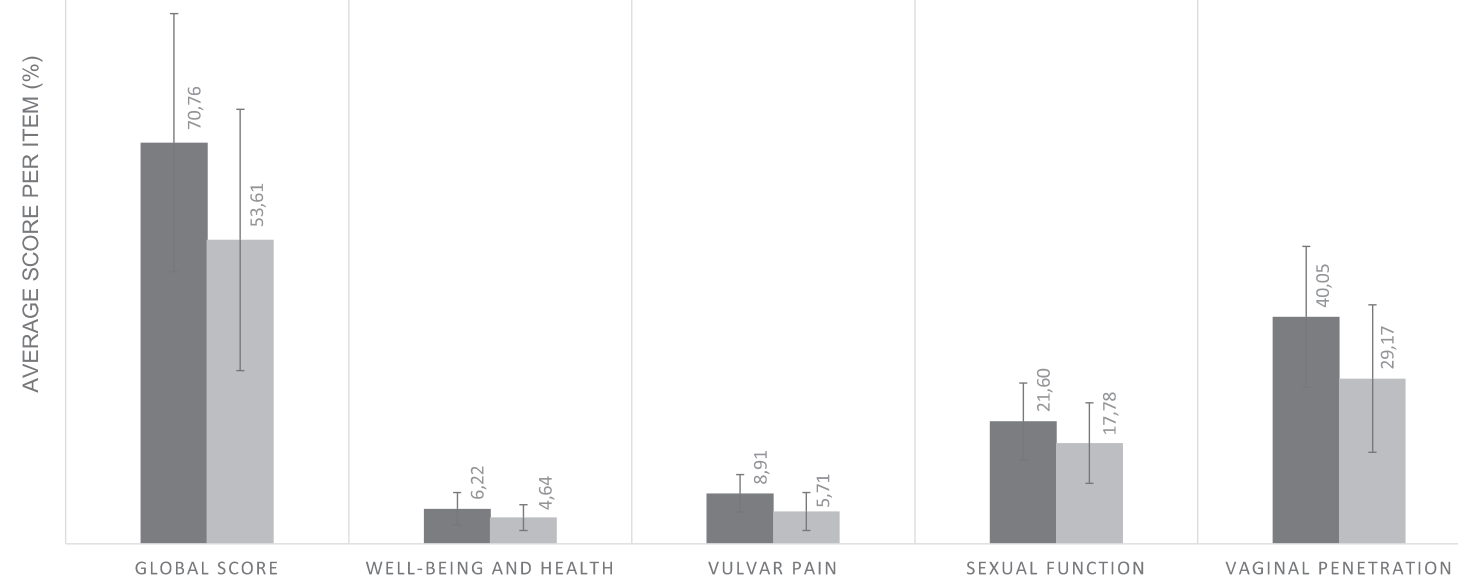

Fig. 1 Perceived global score per item before and after treatment. The scores of perceived general well-being and health, perceived vulvar pain, perceived sexual function and perceived vaginal penetration were significantly improved after treatment. The means of each item are indicated for pre and posttreatment.

three times a day (2\%), with the majority massaging the area once a day (38\%) (Fig. 2).

\section{Opinions and feelings about the treatment}

The question "What do you think about treatment?" was included in Q2. To this, $24 \%$ of the sample answered that physical treatment was extremely helpful $(n=11), 49 \%(n=22)$ stated that it helped very much, and $22 \%(n=10)$ felt it was moderately helpful. None of them stated that the treatment did not help them at all (Fig. 3).

\section{DISCUSSION}

To our knowledge, this is the first study to suggest the exclusive use of manual perineal rehabilitation with lidocaine $2 \%$ gel for the treatment of vulvodynia in women. Many studies have evaluated the effect of physiotherapy on vulvodynia using biofeedback, electrostimulation and/or PFMT [15], transcutaneous electrical nerve stimulation [34], and recently extracorporeal shock wave therapy [13]. Other studies concern medical treatment, psychological treatment or vestibulectomy [15].

Recovering the functionality of the pelvic floor, in term of awareness, contraction, relaxation, and normal tone, is one of the most important points of physical treatment. According to other studies [35, 36], the present one used both active PFMs contraction and active PFMs relaxation to achieve a reduction in tone and an improvement of the awareness of the pelvic floor [35-37]. Likewise, some authors added the simultaneous intervention of global muscle relaxation and breathing techniques to their studies $[38,39]$.

Women with PVD show a lower score of perception of general health compared to controls [37]. Based on the SF-36 results, Naess et al. indicated the perceived impact of PVD on these women's lives in their study [37]. They highlighted the need to learn techniques to control the fear of pain, decrease the apprehension of a future attempt of vaginal penetration, and manage the pain-coping strategies $[37,40]$, which is a reason for including global relaxation and breathing techniques in the protocol of treatment. And because higher rates of sexual dysfunctions have been reported among women with PVD [17], psychoeducation on sexuality was delivered by the physiotherapist during the sessions. Vaginal dilators were not used to dilate the vagina but to regain self-confidence for the resumption of possible sexual intercourse. Patients were suggested to avoid vaginal penetration before this step of the treatment. Talking about foreplay and sexual positions and advising patients to apply lidocaine $2 \%$ gel before intercourse were a crucial part of the treatment $[2,25]$.

Given that sensitisation of the peripheral vestibular nerves has been suggested as a possible mechanism of the pain in PVD [30], local anaesthetics could induce desensitisation of the vestibular area. Lidocaine ointment $5 \%$ is the most commonly recommended local medication $[2,19,41]$. In this study, lidocaine $2 \%$ gel was preferred due to previous reports of patients experiencing severe sensation of vulvar burning after the application of the lidocaine ointment $5 \%$. Statistically, the manual perineal rehabilitation using lidocaine $2 \%$ gel had a positive impact on all the items. Regular sessions and frequent self-massage using lidocaine $2 \%$ gel reinforced the effectiveness of the treatment. It was observed that patients who continued massaging during the first COVID-19 lockdown had better results than those who gave up.

Pukall et al. reported in their review that at that time of publication, no single validated questionnaire existed that covered all the recommended domains for PVD clinical trials [26]. But Dargie et al. developed a multidimensional assessment questionnaire in their research-the Vulvar Pain Assessment Questionnaire (VPAQ), which concerns pain characteristics, emotional/ cognitive functioning, physical functioning, coping skills, and partner factors [42]. A limitation of this study is that more appropriate scales and questionnaires could have been used; for example, the VPAQ, the Goetsch scale to assess subjective pain by the cotton-swab test, or the Female Sexual Distress Scale to evaluate sexually related personal distress in women $[26,43]$.

In the present study, a single physiotherapist carried out the treatments. This may lead to bias by installing trust and sympathy towards the experimenter and thus distorting the study results. This was reported in the responses to the open-ended questions in Q2 about perceived improvement as a result of the treatment. Some patients specified that treatment helped a lot at the beginning but stagnated after a few sessions, that it was emotionally difficult and not pleasant. Nevertheless, others stated that treatment was very beneficial and pain during intercourse was greatly reduced or even disappeared. Also, various patients added that they felt their discomfort was understood and felt less alone in their suffering.

Alternatively, since the reproducibility of the experienced therapeutic gesture is applicable, it could be a proof of the robustness of the study. Another strength of the study is that the 


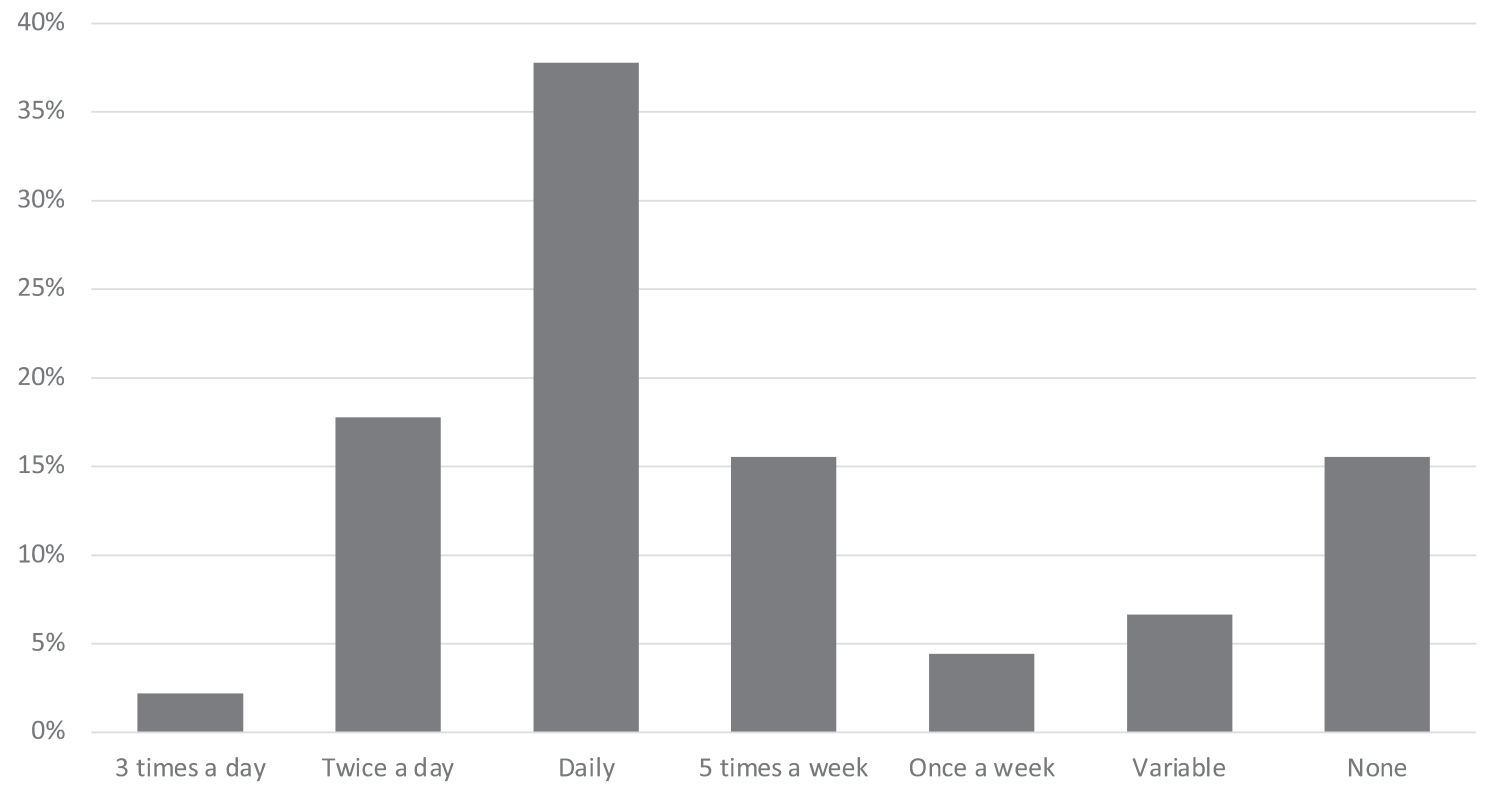

Fig. 2 Representation of the practice and frequency of self-massage with lidocaine $\mathbf{2} \%$ gel. $84 \%$ of the sample practiced self-massage, out of which $38 \%$ applied it once a day.

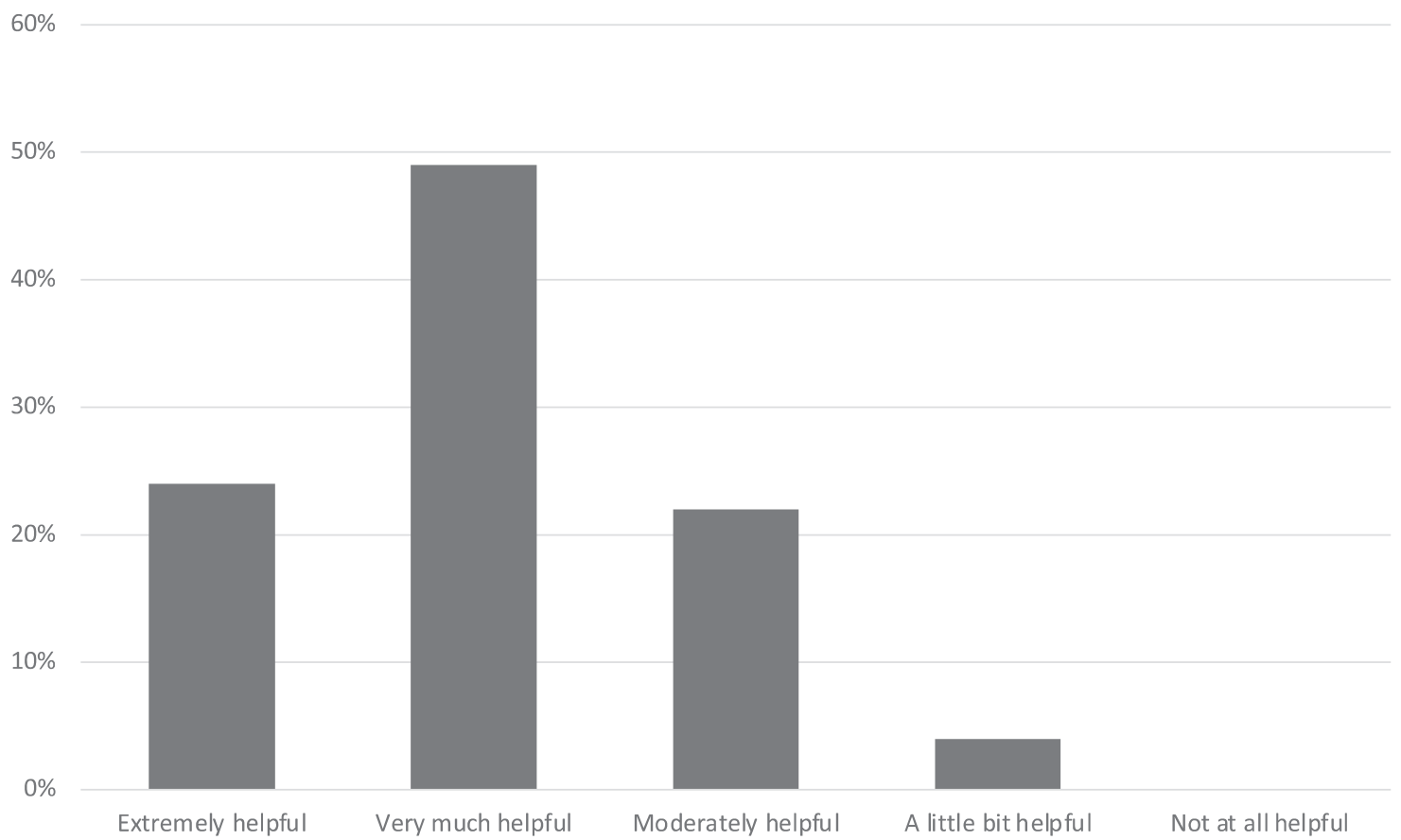

Fig. 3 Distribution of subjects' responses on treatment effectiveness. Physical therapy with lidocaine $2 \%$ gel improved $95 \%$ of the sample ( $24 \%$ extremely; $49 \%$ very much; $22 \%$ moderately). $5 \%$ felt it was a little bit helpful, and none of them stated that it did not help them at all.

treatment was patient-dependent according to the individual case and not the personal preference of a health care provider.

Not being able to identify a reason of their vulvar pain, women with PVD are often very affected [33]. Based on the open-ended questions of Q2, it appears that naming this pain allowed the patients to make progress in resolving this condition.

Women presenting with vulvodynia often experience medical nomadism before receiving the diagnosis [3, 4, 6, 44, 45]. American researchers showed that $60 \%$ of women with PVD consulted three or more specialists without reaching a diagnosis [2]. This confirms the results as more than half of the patients were diagnosed by the experimenter himself. And almost all others received the diagnosis by the same gynaecologist specialised in vulvar pain, which is not representative of healthcare providers. In addition, by analysing the medical prescriptions of the sample included in this study, we found that the diagnosis of PVD is often confused with that of vaginismus. And, depending on the time taken to get the diagnosis, the duration of genital pain lasted from a few months to 21 years, and the number of specialists seen ranged from 1 to 9 .

The women included in the sample who reported pain before sex might have confused it with the apprehension of pain [40]. 
This can probably be explained by negative emotional feelings about sexual activity despite high satisfaction in the relationship. In this study, partners are often very understanding, caring, and patient. Thus, it would be interesting to study the impact of the partner on the efficacity of the treatment.

Finally, our present study was not a randomised controlled trial (RCT), and there is a need for robust and well-designed RCTs to establish the effect of an unindividualised physiotherapy treatment in women with PVD. With this in mind, future work could include a placebo or comparison group in studies on this subject of treatment of vulvodynia. However, given the lack of RCTs, it remains unconclusive whether multimodal approaches are more effective than single treatments [46]. Furthermore, it could be interesting to evaluate the effects of the said treatment at 6 months or 1 year post-treatment.

\section{CONCLUSION}

Exclusive manual perineal rehabilitation using lidocaine $2 \%$ gel seems to be a safe and effective treatment option for provoked vestibulodynia in women in addition to self-massages, significantly improving vulvar pain, sexual function and quality of life of women with PVD.

Vulvodynia puts a significant onus on affected women, their intimate partners, and the healthcare system [33]. To date, there is no standardised, effective, and isolated physiotherapy treatment protocol in connection with PVD [33]. Therefore, the aim of this study was to present a protocol of physical treatment for women suffering from vulvodynia.

\section{REFERENCES}

1. Haefner HK. Report of the International Society for the Study of Vulvovaginal Disease Terminology and Classification of Vulvodynia. J Low Genit Tract Dis. Published online 2007. https://doi.org/10.1097/01.lgt.0000225898.37090.04.

2. Haefner HK, Collins ME, Davis GD, et al. The Vulvodynia Guideline. J Low Genit Tract Dis. 2005;9:40-51. https://doi.org/10.1097/00128360-200501000-00009.

3. Harlow BL, Stewart EG. A population-based assessment of chronic unexplained vulvar pain: have we underestimated the prevalence of vulvodynia? J Am Med Women's Assoc. 1972. 2003;58:82-8.

4. Arnold LD, Bachmann GA, Rosen R, Rhoads GG. Assessment of vulvodynia symptoms in a sample of US women: a prevalence survey with a nested casecontrol study. Am J Obstet Gynecol. 2007;196:128.e1-6. https://doi.org/10.1016/j. ajog.2006.07.047.

5. Lavy RJ, Hynan LS, Haley RW. Prevalence of vulvar pain in an urban, minority population. J Reprod Med. 2007;52:59-62.

6. Reed BD, Crawford S, Couper M, Cave C, Haefner HK. Pain at the vulvar vestibule: a web-based survey. J Low Genit Trac Dis. 2004;8:48-57. https://doi.org/10.1097/ 00128360-200401000-00011.

7. Reed BD, Haefner HK, Sen A, Gorenflo DW. Vulvodynia incidence and remission rates among adult women: a 2-year follow-up study. Obstet Gynecol. 2008;112:231-7. https://doi.org/10.1097/AOG.0b013e318180965b.

8. Bornstein J, Preti M, Simon JA, et al. Descriptors of vulvodynia: a multisocietal definition consensus (International Society for the Study of Vulvovaginal Disease, the International Society for the Study of Women Sexual Health, and the International Pelvic Pain Society). J Low Genit Tract Dis. Published online 2019. https:// doi.org/10.1097/LGT.0000000000000461.

9. Khandker M, Brady SS, Vitonis AF, MacLehose RF, Stewart EG, Harlow BL. The influence of depression and anxiety on risk of adult onset vulvodynia. J Women's Health. 2011;20:1445-51. https://doi.org/10.1089/jwh.2010.2661.

10. Ponte M, Klemperer E, Sahay A, Chren MM. Effects of vulvodynia on quality of life. J Am Acad Dermatol. 2009;60:70-6. https://doi.org/10.1016/j.jaad.2008.06.032.

11. Xie Y, Shi L, Xiong X, Wu E, Veasley C, Dade C. Economic burden and quality of life of vulvodynia in the United States. Curr Med Res Opin. 2012;28:601-8. https://doi. org/10.1185/03007995.2012.666963.

12. Iglesias-Rios L, Harlow SD, Reed BD. Depression and posttraumatic stress disorder among women with vulvodynia: evidence from the population-based woman to woman health study. J Women's Health. 2015;24:557-62. https://doi.org/10.1089/ jwh.2014.5001.

13. Hurt K, Zahalka F, Halaska M, Rakovicova I, Krajcova A. Extracorporeal shock wave therapy for treatment of vulvodynia: a prospective, randomized, double-blind, placebo-controlled study. Eur J Phys Rehabil Med. Published online 2020. https:// doi.org/10.23736/S1973-9087.20.05903-1.

14. De Andres J, Sanchis-Lopez N, Asensio-Samper JM, et al. Vulvodynia-an evidence-based literature review and proposed treatment algorithm. Pain Pract. Published online 2016. https://doi.org/10.1111/papr.12274.

15. Morin M, Carroll MS, Bergeron S. Systematic review of the effectiveness of physical therapy modalities in women with provoked vestibulodynia. Sex Med Rev. Published online 2017. https://doi.org/10.1016/j.sxmr.2017.02.003.

16. Bornstein J, Goldstein AT, Stockdale CK, et al. 2015 ISSVD, ISSWSH and IPPS consensus terminology and classification of persistent vulvar pain and vulvodynia. Obstet Gynecol. 2016;127:745-51. https://doi.org/10.1097/AOG.0000000000001359.

17. Pukall CF, Goldstein AT, Bergeron $S$, et al. Vulvodynia: definition, prevalence, impact, and pathophysiological factors. J Sex Med. 2016;13:291-304. https://doi. org/10.1016/j.jsxm.2015.12.021.

18. Wesselmann U, Bonham A, Foster D. Vulvodynia: current state of the biological science. Pain. 2014;155:1696-701. https://doi.org/10.1016/j.pain.2014.05.010.

19. Stockdale CK, Lawson HW. Vulvodynia guideline update. J Low Genit Tract Dis. 2013. Published online 2014. https://doi.org/10.1097/LGT.0000000000000021.

20. Morin M, Bergeron S, Khalifé S, Mayrand M, Binik YM. Morphometry of the pelvic floor muscles in women with and without provoked vestibulodynia using 4D ultrasound. J Sex Med. 2014;11:776-85. https://doi.org/10.1111/jsm.12367.

21. Gentilcore-Saulnier E, McLean L, Goldfinger C, Pukall CF, Chamberlain S. Pelvic floor muscle assessment outcomes in women with and without provoked vestibulodynia and the impact of a physical therapy program. J Sex Med. 2010;7:1003-22. https://doi.org/10.1111/j.1743-6109.2009.01642.x.

22. Reissing $E$, Brown $C$, Lord M, Binik $Y$, Khalifé S. Pelvic floor muscle functioning in women with vulvar vestibulitis syndrome. J Psychosom Obstet Gynecol. 2005;26:107-13. https://doi.org/10.1080/01443610400023106.

23. Morin M, Binik YM, Bourbonnais D, Khalifé S, Ouellet S, Bergeron S. Heightened pelvic floor muscle tone and altered contractility in women with provoked vestibulodynia. J Sex Med. Published online 2017. https://doi.org/10.1016/j. jsxm.2017.02.012.

24. Glazer HI, Jantos M, Hartmann EH, Swencionis C. Electromyographic comparisons of the pelvic floor in women with dysesthetic vulvodynia and asymptomatic women. J Reprod Med. 1998;43:959-62.

25. Mandal D, Nunns D, Byrne $M$, et al. Guidelines for the management of vulvodynia: guidelines for the management of vulvodynia. Br J Dermatol. 2010;162:1180-5. https://doi.org/10.1111/j.1365-2133.2010.09684.x.

26. Pukall CF, Bergeron S, Brown C, Bachmann G, Wesselmann U. Recommendations for self-report outcome measures in vulvodynia clinical trials. Clin J Pain. Published online 2017. https://doi.org/10.1097/AJP.0000000000000453.

27. Rosen, C Brown, J Heiman, S Leib R. The Female Sexual Function Index (FSFI): a multidimensional self-report instrument for the assessment of female sexual function. J Sex Marital Ther. 2000;26:191-208. https://doi.org/10.1080/ 009262300278597.

28. Rosen NO, Bergeron S, Pukall CF. Recommendations for the study of vulvar pain in women, Part 1: review of assessment tools. J Sex Med. Published online 2020. https://doi.org/10.1016/j.jsxm.2019.10.023.

29. Bergeron S, Brown C, Lord MJ, Oala M, Binik YM, Khalifé S. Physical therapy for vulvar vestibulitis syndrome: a retrospective study. J Sex Marital Ther. 2002;28:183-92. https://doi.org/10.1080/009262302760328226.

30. Bohm-Starke N. Medical and physical predictors of localized provoked vulvodynia. Acta Obstet Gynecol Scand. 2010;89:1504-10. https://doi.org/10.3109/ 00016349.2010 .528368 .

31. Pazmany E, Ly HG, Aerts $L$, et al. Brain responses to vestibular pain and its anticipation in women with genito-pelvic pain/penetration disorder. Neurolmage Clin. Published online 2017. https://doi.org/10.1016/j.nicl.2017.07.017.

32. Sutton KS, Yessick LR, Wild CJ, Chamberlain SM, Pukall CF. Exploring the neural correlates of touch and pain in women with provoked vestibulodynia. Pain. Published online 2020. https://doi.org/10.1097/j.pain.0000000000001778.

33. Vasileva P, Strashilov SA, Yordanov AD. Aetiology, diagnosis, and clinical management of vulvodynia. Przeglad Menopauzalny. Published online 2020. https:// doi.org/10.5114/pm.2020.95337.

34. Murina F, Bianco V, Radici G, Felice R, Di Martino M, Nicolini U. Transcutaneous electrical nerve stimulation to treat vestibulodynia: a randomised controlled trial. BJOG Int J Obstet Gynaecol. Published online 2008. https://doi.org/10.1111/ j.1471-0528.2008.01803.x.

35. Naess I, Bø K. Can maximal voluntary pelvic floor muscle contraction reduce vaginal resting pressure and resting EMG activity? Int Urogynecol J. Published online 2018. https://doi.org/10.1007/s00192-018-3599-1.

36. Backman H, Widenbrant M, Bohm-Starke N, Dahlöf LG. Combined physical and psychosexual therapy for provoked vestibulodynia-an evaluation of a multidisciplinary treatment model. J Sex Res. 2008;45:378-85. https://doi.org/10.1080/ 00224490802398365. 
37. Næss I, Frawley HC, Bø K. Motor function and perception of health in women with provoked vestibulodynia. J Sex Med. 2019;16:1060-7. https://doi.org/10.1016/j. jsxm.2019.04.016.

38. Goldfinger CA. The effectiveness of pelvic floor physiotherapy in women with provoked vestibulodynia. ProQuest Diss Theses. Published online 2008.

39. Goldstein AT, Pukall CF, Brown C, Bergeron S, Stein A, Kellogg-Spadt S. Vulvodynia: assessment and treatment. J Sex Med. 2016;13:572-90. https://doi.org/ 10.1016/j.jsxm.2016.01.020.

40. Benoit-Piau J, Bergeron S, Brassard A, et al. Fear-avoidance and pelvic floor muscle function are associated with pain intensity in women with vulvodynia. Clin J Pain. 2018;34:804-10. https://doi.org/10.1097/AJP.0000000000000604.

41. Zolnoun DA, Hartmann KE, Steege JF. Overnight $5 \%$ lidocaine ointment for treatment of vulvar vestibulitis. Obstet Gynecol. 2003;102:84-7. https://doi.org/ 10.1016/s0029-7844(03)00368-5.

42. Dargie E, Holden RR, Pukall CF. The Vulvar Pain Assessment Questionnaire inventory. Pain. 2016;157:2672-86. https://doi.org/10.1097/j.pain.0000000000000682.

43. Derogatis LR, Rosen R, Leiblum S, Burnett A, Heiman J. The Female Sexual Distress Scale (FSDS): initial validation of a standardized scale for assessment of sexually related personal distress in women. J Sex Marital Ther. 2002;28:317-30. https:// doi.org/10.1080/00926230290001448.

44. Prendergast SA. Pelvic floor physical therapy for vulvodynia. Obstet Gynecol Clin North Am. 2017:44:509-22. https://doi.org/10.1016/j.ogc.2017.05.006.

45. Lindström S, Kvist LJ. Treatment of provoked Vulvodynia in a Swedish cohort using desensitization exercises and cognitive behavioral therapy. BMC Women's Health. Published online 2015. https://doi.org/10.1186/s12905-015-0265-3.

46. Bergeron S, Reed BD, Wesselmann U, Bohm-Starke N. Vulvodynia. Nat Rev Dis Primer. 2020;6:36. https://doi.org/10.1038/s41572-020-0164-2.

\section{ACKNOWLEDGEMENTS}

The authors are very grateful to Manon Blasco for her help with the statistical analysis.

\section{AUTHOR CONTRIBUTIONS}

AC: investigator, experimenter and principal author. MC: methodology of the paper and reviewer. VA: methodology of the study, principal prescriber of the sample and reviewer specialised in the subject. GV: methodology of the study and reviewer specialised in the subject.

\section{COMPETING INTERESTS}

The authors declare no conflict of interest.

\section{ADDITIONAL INFORMATION}

Correspondence and requests for materials should be addressed to A. Close.

Reprints and permission information is available at http://www.nature.com/ reprints

Publisher's note Springer Nature remains neutral with regard to jurisdictional claims in published maps and institutional affiliations. 